\title{
Validez factorial del cuestionario de expectativas hacia el consumo de alcohol (AEQ -III), en adultos de Bucaramanga, Colombia
}

\author{
O scar F Herrán1,2,a, María F Ardila2,b. \\ Factorial validation of the alcohol \\ expectancy questionnaire (AEQ -III), in \\ adults living in Bucaramanga, Colombia
}

Background: Alcohol consumption is associated to expectancies of global positive behavioral changes, improvement in sexual performance and a higher level assertiveness. These issues are evaluated by the Alcohol Expectancy Questionnaire (AEQ-III). Aim: To perform a factorial validation of the Alcohol Expectancy Questionnaire (AEQ-III). Materials and methods: The questionnaire was applied to a representative sample of 601 subjects aged 18 to 60 years (334 males). These subjects also answered a socioeconomic questionnaire. The significant variables of the questionnaire were selected using an exploratory factorial analysis. Based on the selected variables, the verification was performed using multiple models of structural equations. Four factors, namely "decrease in physical tension", "decrease in psychological tension", "increase in expressivity and sexuality" and "inhibition and feelings of power", were identified as significant. Results: The factorial solution explains $59 \%$ of the variance. All the factors are conceptually and statistically consistent, with a Cronbach alpha ranging between 0.75 and 0.83 . The global internal consistency was of 0.85 . Pearson correlation coefficient among factors ranged between 0.45 and 0.74 . There was a significant difference among socioeconomic levels for the factors "lack of inhibition and feelings of power" and "decrease of psychological tension". Goodness of fit statistics and parsimony were high, ranging from 0.80 to 0.93 . Conclusions: The expectancy "decrease of physical tension" and "decrease of psychological tension" are a part of individual behavior and could predict problem drinking. The factors "increase of expressiveness and sexuality" and "lack of inhibition and feelings of power", are more associated to group behaviors (Rev Méd Chile 2007; 135: 488-95).

(Key words: Alcohol drinking; Alcoholism; Factor analysis, statistical)

\footnotetext{
Recibido el 27 de marzo, 2006. Aceptado el 14 de julio, 2006.

${ }^{1}$ Escuela de Nutrición y Dietética, Universidad Industrial de Santander. ${ }^{2}$ Observatorio Epidemiológico de Enfermedades Cardiovasculares, Centro de Investigaciones Epidemiológicas, Universidad Industrial de Santander, Bucaramanga, Colombia.

${ }^{a}$ Nutricionista Dietista. MSc. Epidemiología

bEnfermera. MSc. Epidemiología
}

Correspondencia a: Oscar Fernando Herrán Falla. Calle 33

Número 29-31, apartamento 501. Zona Postal 3001. A.A.

2604. Bucaramanga, Colombia, Sur América. TeleFax: (57-

7) 6345781. Teléfono: (57-7) 6320715.

E mail: herran28@intercable.net.co / herran@uis.edu.co 
$\mathrm{E}^{1}$ consumo de alcohol (CA) y los problemas relacionados han aumentado en Colombia durante la última década ${ }^{1}$. Más de $90 \%$ de la población adulta consume bebidas alcohólicas y 14\% es alcohólica, según la prueba CAGE (Cutting down, Annoyance by criticism, Gulty feeling, and Eye-openers) ${ }^{2,3}$. A pesar de la necesidad de estudiar la etiología del CA, en Bucaramanga y Colombia no existen investigaciones sobre los determinantes del CA, del abuso o la dependencia. Las encuestas nacionales sobre CA y psicoactivos afirman que las variables asociadas a las redes de apoyo, la valoración social del CA y de las bebidas alcohólicas, y otras complejas de medir, son determinantes del CA y del riesgo de alcoholismo y recomiendan el estudio prioritario de éstas ${ }^{4,5}$. Un estudio realizado en Bucaramanga reportó que ninguna variable biológica, sociodemográfica o dietaria estuvo asociada al $\mathrm{CA}^{2}$.

El CA y los problemas relacionados, históricamente, han sido explicados desde los desórdenes de la personalidad, hasta diferentes teorías como la del aprendizaje social, la del desarrollo, la teoría cognitiva, la del aprendizaje, la de respuesta emocional y de la influencia genética ${ }^{6}$. Una aproximación psicosocial llamada teoría de las expectativas, es otro de los mecanismos explicatorios del CA que incorpora muchos de los elementos de las teorías clásicas, pero con una visión reduccionista desde la perspectiva de la neurobiología 6 .

El concepto de expectativa fue relacionado con el consumo de alcohol por primera vez en $1932^{6}$, pero sólo hasta hace dos décadas y debido a los avances en el campo de la neurobiología de las adicciones, se ha consolidado como una teoría capaz de predecir el CA en población adolescente y adulta ${ }^{6}$. Esta aproximación, también llamada de mecanismos de aprendizaje primitivos y habituales, toma aspectos de la memoria, de la motivación y las emociones de los sujetos y enfatiza sobre la capacidad de éstos para usar información genética o del ambiente para preparar la adaptación del comportamiento a circunstancias tardías ${ }^{7}$. Las expectativas son, además, un concepto psicosocial clave en la explicación del comportamiento del CA y del abuso ${ }^{8}$. Redefinidas en 1980 por Brown y Goldman; "este constructo refleja la representación en la memoria de la información adquirida por un individuo alrededor de comportamientos ciertos dentro de contextos generales y específicos. Estas expectativas pueden ser adquiridas a través de experiencias directas, o de consecuencias observadas o de otras muchas formas en que los humanos adquieren el conocimiento. Esta memoria a su turno estructura la percepción e interpretación del ambiente y por lo tanto determinan el comportamiento"9.

El cuestionario de expectativas hacia el alcohol (AEQ-III) es el más usado cuando se estudian las expectativas hacia el $\mathrm{CA}^{10}$. El objetivo del presente estudio es hacer la adaptación del AEQ-III a esta cultura y establecer su validez factorial en una muestra de adultos, residentes en el área urbana de Bucaramanga, Colombia.

\section{Material y MÉTOdo}

Bucaramanga es una ciudad de clima cálido $\left(26^{\circ} \mathrm{C}\right)$, cuenta con 800.000 habitantes y es la más desarrollada de la región nororiental de Colombia. Durante 2005 se desarrolló un estudio de corte transversal.

Tamaño de la muestra. Seiscientos un sujetos fueron encuestados. El tamaño muestral fue definido como cinco por el número de ítems del AEQIII $(5 \times 120)$. Cinco es el mínimo de sujetos por ítem recomendado para establecer la validez factorial de un cuestionario ${ }^{11,12}$.

Selección de sujetos. Los sujetos fueron seleccionados a través de muestreo multietápico para representar por sexo y estrato socioeconómico a la población. Los que cumplieron los siguientes criterios fueron elegibles: 1- Entre 18 y 60 años. 2Residir en la ciudad dos años o más. 3- No asistir a un grupo de apoyo como alcohólicos anónimos y 4- Que manifestaran su deseo de participar suministrando voluntariamente la información. De un mapa de Bucaramanga, fueron elegidas al azar manzanas y luego casas por estrato socioeconómico, en cada vivienda se realizó un censo de elegibles y aleatoriamente se seleccionó uno por vivienda. Si esta persona se rehusaba, se elegía otra persona de la misma vivienda. Después de seleccionarlos, de informarlos sobre los objetivos, de garantizar la confidencialidad y de obtener un consentimiento escrito, se encuestaron. El proyecto $\mathrm{y}$ los procedimientos fueron aprobados por el 
comité de ética en investigación de salud de la Universidad Industrial de Santander.

Fuentes de información. Las fuentes de información fueron primarias. Dos encuestas fueron aplicadas mediante entrevista directa; un cuestionario sobre aspectos sociodemográficos y el AEQ-III ${ }^{10}$.

AEQ-III es el cuestionario más usado cuando se estudian las expectativas hacia el consumo de alcohol, fue diseñado en 1980 y perfeccionado hasta 1987 por Goldman M, Brown S, y Christiansen $\mathrm{BA}^{8,10}$, los encuestadores no necesitan de entrenamiento específico y sus 120 ítems pueden responderse entre 20 y 30 min por autorreporte 0 entrevista directa. AEQ-III evalúa las esperanzas (expectativas) que determinan el consumo de alcohol y mide el grado en que un individuo espera una variedad de efectos generales y específicos al consumir alcohol. AEQ-III cuenta con estudios de reproducibilidad, coeficiente $r=0,64$ (test-retest) y una consistencia interna entre $0,72 \mathrm{a}$ $0,92^{6,9}$. Además, cuenta con estudios de validez de criterio (concurrente y predictiva) y sus resultados son estables cuando son llevados a escalas universales $6,8,9,13-17$. El formato de respuesta es dicotómico. AEQ-III evalúa las expectativas hacia seis factores ${ }^{9}$ : 1- Cambios globales positivos con la experiencia de consumo, 2- La mejoría en el desempeño sexual, 3- El aumento de placer social y físico, 4- La asertividad social (afirmación en el grupo), 5- La reducción de la tensión física y 6- La excitación o reafirmación del poder interpersonal (sensación de liderazgo o agresividad). La versión del AEQ-III que fue aplicada es la III para población adulta, ésta fue retro-traducida al español-inglés de manera independiente por dos expertos, con base en la última versión original desarrollada en $1987^{9}$. La adecuación a la jerga de Bucaramanga se hizo con base en la versión mexicana $^{6,18}$ y con base en un estudio piloto realizado en 60 adultos en la población blanco (diez por estrato socioeconómico).

Estadística. Análisis factorial exploratorio. Este análisis fue conducido en dos niveles, en cada uno fue derivada una solución factorial (modelos A y B). A pesar de que con un tamaño muestral como el utilizado en este estudio $(n=601)$, es posible obtener significancia estadística con cargas factoriales de 0,21 (alfa $=0,01)^{11,19}$, como criterios de selección de variables fue utilizado un valor de 0,40 conjuntamente con una comunalidad mínima de 0,40 (1 - Uniqueness).

El primer nivel fue realizado con los 120 ítems del AEQ-III con base en la matriz de covarianza y utilizando una rotación PROMAX, debido a que los factores latentes (expectativas) están correlacionados $^{19}$. Coherente con los conceptos subyacentes en el AEQ-III, la exploración inició con seis factores y se repitió para cinco, cuatro, tres y dos. En cada solución factorial las variables fueron agrupadas con base en la mayor carga factorial, se crearon nuevas bases de datos con ellas y nuevamente realizado el procedimiento. Para verificar la pertinencia de la reducción de variables se calculó el estadístico Kaiser-Meyer-Olkin (KMO) ${ }^{19,20}$.

El segundo nivel del análisis factorial fue realizado sólo en un grupo de variables. Los criterios que definieron la elección de éstas, fueron el porcentaje de la varianza explicada, el número de variables observadas que se mantenía al aplicar los criterios de reducción, el número de variables latentes (factores o expectativas) y el resultado del estadístico KMO, que fue calculado para las variables seleccionadas, así como para los factores extraídos.

El subgrupo de variables que obtuvo el mejor balance, entre la varianza explicada, el número de factores y el estadístico $\mathrm{KMO}$, fue reagrupado con base en las dimensiones subyacentes del AEQ-III. Múltiples arreglos fueron valorados a través del diseño de modelos de ecuaciones estructurales $(\mathrm{MEE})^{19}$. Con el fin de comparar el modelo final (modelo C) con los modelos A y B, fueron diseñados MEE y calculados diferentes índices de bondad de ajuste; razón de verosimilitud $\left(\mathrm{X}^{2}\right)$, error de aproximación cuadrático medio (RMSEA), el índice Tucker-Lewis (NNFI), de ajuste normal (NFI), de bondad de ajuste (GFI) y el índice de ajuste de parsimonia $(\mathrm{PNFI})^{19}$. Todas las soluciones factoriales, fueron realizadas con la matriz de covarianza y la estimación de la máxima verosimilitud (likelihood) ${ }^{19-21}$. Para facilitar la comparación, los puntajes en los factores fueron escalas aditivas ${ }^{6,18}$. En los tres modelos, la consistencia interna intra factor y entre factores, fue determinada con el alfa de Cronbach $(\mathrm{AC})^{3,22}$. Con el coeficiente $r$ de Pearson, se estableció la correlación entre factores.

Toda la información fue sometida a doble digitación y validada en EpiInfo $\mathrm{v} 6.04 \mathrm{~d}^{23}$. La 
descripción de las variables sociodemográficas fue realizada con medidas descriptivas apropiadas e intervalos de confianza del 95\% (IC). Los estratos socioeconómicos 1 y 2, los más bajos en la escala de ingreso y desarrollo, se agruparon en el nivel socioeconómico 1, los 3 y 4 en el nivel 2 y los estratos 5 y 6 en el nivel 3. Para determinar si existía tendencia lineal en los puntajes de las expectativas a través de las categorías en las variables sociodemográficas se utilizó el método de Cuzick $^{24}$. Pruebas t de student y ANOVA fueron realizadas para evaluar si existían diferencias por sexo, la escolaridad o la edad. El manejo de variables y los cálculos estadísticos, fueron realizados con STATA/SE ${ }^{25}$. Los MEE fueron realizados con Lisrel ${ }^{26}$.

\section{Resultados}

Población estudiada. Un total de 1.095 casas fueron visitadas, 2.655 sujetos fueron declarados elegibles, de ellos 601 fueron encuestados. Cien sujetos participaron por cada uno de los seis estratos socioeconómicos originales. El 55,6\% fueron hombres. El 1,7\% no terminó la primaria. El $4,0 \%$, al momento de la encuesta, tenía un diagnóstico médico relacionado con alteraciones metabólicas o gastrointestinales. La edad media fue de 34,7 años, IC 33,8 a 35,6, sin diferencia por sexo $(p=0,070)$. La escolaridad media fue de 12,8 años, IC 12,5 a 13,1 sin diferencia por sexo ( $p$ $=0,955)$, pero sí por el nivel socioeconómico ( $p$ $\leq 0,001)$. Otras características de la población estudiada se presentan en la Tabla 1.

Análisis exploratorio. La solución factorial escogida en el primer nivel fue con veintitrés variables, agrupadas en tres factores, ellas explican $59 \%$ de la varianza. Un KMO mayor de 0,80 justifica una reducción de datos. El KMO para las variables $(0,91)$ justificó su agrupación, de la misma manera el KMO justificó los tres factores $(0,70)$ (Tabla 2). La Tabla 3, ilustra la agrupación de las variables como resultado del análisis de segundo nivel (modelo B) y de orden superior (modelo C). La afirmación "el alcohol parece ser mágico", fue eliminada del modelo C por ser inespecífica y su alto aporte al error.

La solución factorial final (modelo C), tiene cuatro factores. F1: "incremento de la expresividad y la sexualidad", promedio 2,7, IC 2,6 a 2,8, AC 0,75. F2: "disminución de la tensión física", promedio 3,5, IC 3,3 a 3,6, AC 0,75. F3: "desinhibi-

Tabla 1. C aracterísticas de la población estudiada

\begin{tabular}{|llll|}
\hline Variable & $\begin{array}{l}\text { Total } \\
(\mathrm{n}=601)\end{array}$ & $\begin{array}{l}\text { Hombre } \\
(\mathrm{n}=334)\end{array}$ & $\begin{array}{l}\text { Mujer } \\
(\mathrm{n}=267)\end{array}$ \\
\hline $\begin{array}{l}\text { Grupos de edad (años) } \\
\quad \text { Menor de 25 }\end{array}$ & $110(18,3)$ & $53(15,9)$ & $57(21,4)$ \\
$\quad \begin{array}{l}\text { Entre 25 y 39 } \\
\text { Mayor de 40 }\end{array}$ & $306(50,9)$ & $174(52,1)$ & $132(49,4)$ \\
$\begin{array}{l}\text { Escolaridad (años) } \\
\text { Hasta primaria }\end{array}$ & $185(30,8)$ & $107(32,0)$ & $78(29,2)$ \\
$\begin{array}{l}\text { Entre 6 y 11 } \\
\text { Entre 12 y 16 }\end{array}$ & $28(4,7)$ & $20(6,0)$ & $8(3,0)$ \\
$\begin{array}{l}\text { 17 ó más } \\
\text { Nivel socioeconómico* }\end{array} \quad$ 123 $(36,8)$ & $97(36,3)$ \\
$\quad$ Uno & $220(36,6)$ & $124(37,1)$ & $114(42,7)$ \\
$\quad$ Dos & $115(19,1)$ & $67(20,1)$ & $48(18,0)$ \\
$\quad$ Tres & $200(33,3)$ & $119(35,6)$ & $81(30,3)$ \\
\end{tabular}

*Al nivel uno pertenecen los estratos socioeconómicos 1 y 2; al dos, los estratos 3 y 4; al nivel tres, pertenecen los estratos socioeconómicos 5 y 6. 
Tabla 2. C riterios para la determinación del número de factores y la reducción de variables

\begin{tabular}{|lccccc|}
\hline $\begin{array}{l}\text { No. } \\
\text { Factores }\end{array}$ & $\begin{array}{c}\text { Varianza (\%) } \\
\text { explicada }\end{array}$ & $\begin{array}{c}\text { No } \\
\text { variables }\end{array}$ & $\begin{array}{c}\text { Est. KMO* } \\
\text { variables }\end{array}$ & $\begin{array}{c}\text { Est. KMO* } \\
\text { factores }\end{array}$ & Diferencia \\
\hline 1 & 54 & 21 & 0,95 & & \\
2 & 57 & 26 & 0,94 & 0,50 & 0,44 \\
3 & 59 & 23 & 0,91 & 0,70 & 0,21 \\
4 & 62 & 35 & 0,93 & 0,77 & 0,16 \\
5 & 64 & 34 & 0,94 & 0,86 & 0,08 \\
6 & 67 & 38 & 0,94 & 0,88 & 0,06 \\
\hline
\end{tabular}

*Est. KMO. Estadístico Kaiser-Meyer-Olkin para la pertinencia de la agrupación ${ }^{19}$; para el número de variables seleccionadas y para el número de factores.

Tabla 3. Agrupación de variables: Solución A, análisis exploratorio de primer nivel (tres factores).

Solución B, análisis exploratorio de segundo nivel (tres factores). Solución C, análisis de orden superior o confirmatorio (cuatro factores)

\begin{tabular}{|lrrr|}
\hline & Modelo A & Modelo B & Modelo C \\
Número de variables que da origen & 120 & 23 & 23 \\
Ítem & & & \\
\hline El alcohol hace que me preocupe menos & Factor 1 (F1) & F3 & F4 \\
Unos tragos hacen fácil el hablar con la gente & F1 & F3 & F4 \\
El alcohol disminuye la tensión muscular & F1 & F3 & F2 \\
Después de unos tragos me mejora el genio & F1 & F3 & F4 \\
El alcohol parece ser mágico & F1 & F3 & Eliminada \\
El alcohol me permite ser más positivo & F1 & F3 & F4 \\
Me siento más creativo después de haber tomado & F1 & F1 & F3 \\
Soy mejor amante después de unos tragos & F2 & F1 & F1 \\
Las mujeres son agresivas después de unos tragos & F2 & F1 & F3 \\
Las mujeres hablan más después de unos tragos & F2 & F1 & F1 \\
El alcohol me ayuda a dormir mejor & F2 & F1 & F2 \\
El alcohol me hace sentir mejor físicamente & F2 & F1 & F2 \\
El trago es refrescante después de hacer ejercicio & F2 & F1 & F2 \\
El alcohol aumenta mi intimidad con las personas & F2 & F1 & F1 \\
El alcohol me permite dormir más fácilmente & F2 & F1 & F2 \\
Cuando tomo siento más confianza en mí mismo & F2 & F1 & F1 \\
El alcohol puede transformar mi personalidad & F3 & F2 & F3 \\
Cuando las mujeres toman, son frescas con el sexo & F3 & F2 & F3 \\
En las reuniones sociales, cuando se toma el & & & \\
ambiente es más agradable & F3 & F2 & F3 \\
Cuando estoy tomando, me siento como quiero & F3 & F2 & F3 \\
El alcohol elimina los sentimientos de inferioridad & F3 & F2 & F3 \\
Algunas bebidas alcohólicas me quitan los nervios & F3 & F2 & F3 \\
Cuando tomo, me siento capaz de cualquier cosa & F3 & F2 & F3 \\
\hline
\end{tabular}


ción y sentimientos de poder", promedio 6,4 , IC 6,2 a 6,6, AC 0,83. F4: "disminución de la tensión psicológica", promedio 2,8, IC 2,7 a 2,9, AC 0,78. La consistencia interna global alcanzó un valor de 0,85. En los modelos A y B, igualmente se alcanzó una alta consistencia interna, $\mathrm{AC}$ de 0,82 y 0,80 , respectivamente. La correlación entre $\mathrm{F} 1$ y F2 fue de $r=0,69$, entre $F 1$ y F3 de $r=0,69$, entre $F 1$ y $F 4$ de $r=0,45$, entre $F 2$ y F3 de $r=0,74$, entre $F 2$ y F4 de $r=0,60$ y entre $F 3$ y $F 4$ de $r=0,59$.

Relación entre expectativas y variables sociodemográficas. No se evidenciaron diferencias en ninguno de los cuatro factores por sexo, grupo de edad o nivel de escolaridad. Los factores "desinhibición y sentimientos de poder" y "disminución de la tensión psicológica", fueron diferentes según el nivel socioeconómico ( $p<0,001$ ), en ambos el nivel dos alcanzó el mayor puntaje y el tres el menor. Tampoco se evidenció tendencia lineal entre las expectativas y las variables sociodemográficas.

Evaluación de las soluciones factoriales. El valor de $\mathrm{p}$ para el chi-cuadrado de la razón de verosimilitud en los tres modelos fue menor de 0,05. El valor ideal del RMSEA es por debajo de 0,08. Para los demás índices, un valor de cero refleja un pobre ajuste y un valor de 1 , un ajuste perfecto ${ }^{19,21}$. La Tabla 4 resume estos hallazgos y evidencia el desempeño de las soluciones factoriales presentadas.

\section{Discusión}

Limitaciones y alcance del estudio. Debido al diseño, los resultados son aplicables a hombres y mujeres mayores de 18 años con la cultura hacia el alcohol particular del área urbana de Bucaramanga. Debido a la ausencia de datos demográficos para esta población, no pudimos establecer con certeza si la distribución de la edad en la muestra sigue la de la población general. Sin embargo, la distribución por sexo es similar a la reportada en la encuesta de demografía y salud ${ }^{27}$. La proporción de primaria incompleta 1,7\%, fue menor que la reportada para la población blanco $(4,8 \%)^{27}$. Algunos autores argumentan que la muestra ideal para estudiar la validez factorial de un cuestionario es de diez por ítem, pero aclaran que éste también es necesario en caso de $\mathrm{n}<100$; también afirman que cuando el tamaño de la muestra es $>300$, es adecuado cinco sujetos por item $^{11}$. Nuestro estudio con $\mathrm{n}=601$, es una muestra grande. Además, se utiliza un criterio conservador para alcanzar la significación estadística de las cargas factoriales en la selección de las variables $(0,40 \text { en vez de } 0,21)^{19}$.

Validez factorial. A pesar de eliminar 98 ítems del AEQ-III original, nuestros hallazgos indican que el AEQ-III se adaptó consistentemente en la población adulta. La solución factorial final, modelo C, tiene cuatro dimensiones coherentes, conceptual y estadísticamente (alfa de Cronbach global 0,85).

Tabla 4. Análisis de factores: valores alcanzados en las medidas de bondad de ajuste para tres modelos desarrollados

\begin{tabular}{|c|c|c|c|}
\hline $\begin{array}{l}\text { № de factores (№ de variables) } \\
\text { Estadístico }\end{array}$ & $\begin{array}{l}\text { Modelo A } \\
3 \text { (23) }\end{array}$ & $\begin{array}{l}\text { Modelo B } \\
3 \text { (23) }\end{array}$ & $\begin{array}{l}\text { Modelo C } \\
4(22)\end{array}$ \\
\hline Razón de verosimilitud $\left(\mathrm{X}^{2}\right)^{*}$ & $1.619(227)$ & $1.619(227)$ & $1.680(203)$ \\
\hline Error de aprox. cuadrático medio (RMSEA) & 0,10 & 0,10 & 0,11 \\
\hline Índice Tucker-Lewis (NNFI) & 0,93 & 0,93 & 0,91 \\
\hline Índice de ajuste normal (NFI) & 0,92 & 0,92 & 0,91 \\
\hline Índice de bondad de ajuste (GFI) & 0,81 & 0,81 & 0,80 \\
\hline Índice de ajuste de parsimonia (PNFI) & 0,83 & 0,83 & 0,80 \\
\hline
\end{tabular}

*Para todos valor de $\mathrm{p}<0,05$. 
El factor "desinhibición y sentimientos de poder", fue el más estable durante el análisis factorial. No se encontró evidencia de las seis dimensiones propuestas originalmente ${ }^{9}$, ni de las ocho identificadas en estudiantes universitarios mexicanos ${ }^{6,18}$. Lo anterior puede explicarse de una parte por las variaciones culturales en los grupos sociales y de otra, por la menor edad y mayor nivel educativo en los estudiantes mexicanos. Es bien sabido que el adulto joven aún está explorando sus comportamientos personales y sociales para reafirmarlos o rechazarlos ${ }^{6,9}$

Un valor de p mayor de 0,05 es deseable en la $\mathrm{X}^{2}$ como indicador de buen ajuste en un MEE. Con excepción de la razón de verosimilitud $\left(\mathrm{X}^{2}\right)$, en todas las soluciones factoriales, los índices de ajuste y parsimonia obtuvieron valores altos. Es posible que la asociación entre el nivel socioeconómico y los factores "desinhibición y sentimientos de poder" y "disminución de la tensión psicológica", se explique por la presencia de variaciones en el patrón cultural y la socialización. También es probable que dada la estratificación económica, cambien los significados simbólicos hacia el alcohol y su consumo. Por la naturaleza del presente estudio, no fue posible verificar estas hipótesis.

Utilidad clínica y epidemiológica. Una de las principales estrategias utilizadas en el tratamiento del CA, los problemas relacionados y el alcoholismo, es la intervención a través de un consejero motivador 28,29 . Este tipo de consejería dirigida nació en 1983 y tiene como objetivo primario incentivar o despertar la motivación intrínseca en

\section{REFERENCIAS}

1. Herrán OF, Ardila MF. Validity and Reproducibility of two Semi-Quantitative Alcohol Frequency Questionnaires for Colombian Population. Public Health Nutrition 2005; 8: 181-8.

2. HeRrán OF, ARdila MF. Consumo de alcohol, riesgo de alcoholismo y alcoholismo en Bucaramanga, Colombia, 2002-2003. Colombia Médica 2005; 36: 158-67.

3. Herrán OF, ArdiLA MF. Validez interna y reproducibilidad de la prueba CAGE en Bucaramanga, Colombia. Biomédica 2005; 25: 231-41.

4. TorRES DE GY. Alcohol: Prevalencia de consumo y los sujetos para reconocer y hacer algo sobre sus problemas potenciales o actuales. Este tratamiento, en la clínica ha demostrado una eficacia de $75 \%$ en sujetos que son renuentes al cambio o en quienes son ambivalentes sobre cambiar su comportamiento ${ }^{28,29}$. La motivación también ha demostrado ser útil en el tratamiento grupal de alcohólicos ${ }^{28-30}$. El conocimiento de las expectativas positivas hacia el CA en un grupo social en particular, facilita en la práctica clínica este tipo de intervenciones y anticipa mejores resultados en pacientes alcohol dependientes o con consumo problemático ${ }^{30}$. Además, el conocimiento de las expectativas se constituye en la base para la implementación de otro tratamiento alternativo para el abuso y la dependencia del alcohol, como es la intervención conductual combinada, que suma a la consejería dirigida el uso de fármacos ${ }^{31}$.

En resumen, identificamos cuatro dimensiones en el AEQ-III. Dos de ellas "disminución de la tensión física" y "disminución de la tensión psicológica", hacen parte de la conducta individual, y en futuros estudios podrían predecir el consumo problemático ${ }^{18}$. Las otras dos, "incremento de la expresividad y la sexualidad" y "desinhibición y sentimientos de poder", están más relacionadas con la conducta grupal. Es necesario estudiar el comportamiento de estas escalas en adolescentes y adultos jóvenes, para intentar estrategias de prevención del consumo excesivo. La solución factorial aquí presentada será útil al realizar estudios con el objeto de establecer la relación del AEQ-III con el CA, el consumo problemático y el alcoholismo en adultos.

dependencia en Colombia. Revista de Medicina CES 1999; 12: 1-9.

5. Presidencia de la Republica/Ministerio de Salud/ Programa Rumbos. Estudio nacional sobre uso y abuso de alcohol y consumo de sustancias sicoactivas, Colombia 2000.

6. Mora-Ríos J, Natera G, Vimatorio J, Vimalvazo R. Validez factorial del cuestionario de expectativas hacia el alcohol (AEQ-III) en estudiantes universitarios. Psicología Conductual 2000; 8: 319-28.

7. Eichenbaum $\mathrm{H}$. How does the brain organize memories? Science 1997; 277: 330-2.

8. BRown SA, GoLdMAN MS, INN A, ANDERSON LR. Expectactions of reinforcement from alcohol their 
domain and relation to drinking patterns. J Consult Clin Psychol 1980; 48: 419-26.

9. Goldman MS, Del Boca FK, Darles J. Alcohol expectancy theory: The application of cognitive neuroscience. En: Leonard KE, Blane HT (Editors). Psychological theories of drinking and alcoholism. Second Edition. New York: Guilford Press, 1999; 203-46.

10. Brown SA, Christiansen BA, Goldman MS. The alcohol expectancy questionnaire: an instrument for the assessment of adolescent and adult alcohol expectancies. J Stud Alcohol 1987; 48: 483-91.

11. Norman G, Streiner D. Bioestadística. Barcelona: Editorial Mosby, 1996; 129-48.

12. TAVelia NM. Análisis de los ítems en la construcción de instrumentos psicométricos. México: Editorial Trillas, 1978; 75.

13. Bierut LJ, Dinwiddie SH, Begleiter H, Crowe RR, Heselbrock V, NuRnBerger JI et al. Familial transmission of substance dependence: alcohol, marijuana, cocaine and habitual smoking. Arch Gen Psychiatry 1998; 55: 982-8.

14. BROwN SA. Expectancies versus background in the prediction of college drinking patterns. J Consult Clin Psychol 1985; 53: 123-30.

15. KLINE P. The relation of alcohol expectancies to drinking patterns among alcoholics: Generalization across gender and race. J Stud Alcohol 1990; 56: $177-85$.

16. Golmand MS. The alcohol expectancy concept: Applications to assessment, prevention, and treatment of alcohol abuse. Appl Prev Psychol 1994; 3: 131-44.

17. Leigh BC, StaCy AW. Alcohol outcome expectancies: Scale construction and predictive utility in higher order confirmatory models. Psychol Assess 1993; 5: 216-29.

18. Mora J, Natera G. Expectativas, consumo de alcohol y problemas asociados en estudiantes universitarios de la ciudad de México. Salud Pública Mex 2001; 43: 89-96.
19. Hair JF, Anderson RE, Tatham RL, Black WC. Análisis multivariante. Quinta edición, Madrid: Prentice Hall, 1999.

20. Hamlton LC. Regression with graphics. Belmont, California: Duxbury Press, 1991; 249-88.

21. KunE P. An easy guide to factor analysis. London: Routledge Press, 1994; 157-84.

22. Glem JA, Guem RR. Calculating, interpreting, and reporting Cronbach's alpha coefficient for likert. Type scales. Presented at the Midwest research to practice conference in adult, continuing, and community education. The Ohio State University, Columbus, OH, October 8-10, 2003. Disponible en: www.alumni-osu.org. Acceso el 10 de febrero de 2006.

23. CDC. EpiInfo, versión 6.04d. Epidemiología en ordenadores. Atlanta, Georgia. Enero, 2001.

24. Cuzick J. A Wilcoxon-type test for trend. Stat Med 1985; 4: 87-90.

25. StataCorp. 2005. Stata Statistical Software: Release 9.1. College Station, TX: StataCorp LP.

26. JöRESKOG K, SöRBORM D. LSREL 8.70. Scientific Software International, Inc, 2004.

27. PROFAMILA. Encuesta Nacional de Demografía y Salud. Resumen Región Oriental, 2000. Bogotá, Colombia: PROFAMIIA, 2000.

28. MILER WR, ROLNICK S. Motivational interviewing: Preparing people for change, $2^{\text {nd }}$ Edition, New York: Guilford Press, 2002; 33-42.

29. Rubak S, Sandbaek A, Lauritzen T, Christensen B. Motivational interviewing: a systematic review and meta-analysis. Br J Gen Pract 2005; 55: 305-12.

30. Jhon U, Veltrup C, Driessen M, Wetterling T, Diwng H. Motivational intervention: An individual counselling vs a group treatment approach for alcohol-dependent in patients. Alcohol alcohol 2003; 38: 263-9.

31. Longabaugh R, Zweben A, Locastro JS, Miler WR. Origins, issues and options in the development of the combined behavioral intervention. J Stud Alcohol 2005; Suppl(15): 179-87; discussion 168-9.

Agradecimiento

A Luis C. Orozco, de la Escuela de Enfermería de la Universidad Industrial de Santander por permitirnos usar LISREL ${ }^{26}$. 